PROCEEDINGS OF THE

AMERICAN MATHEMATICAL SOCIETY

Volume 129, Number 4, Pages 943-946

S 0002-9939(00)05613-6

Article electronically published on October 16, 2000

\title{
TRACE METHODS IN TWISTED GROUP ALGEBRAS
}

\author{
D. S. PASSMAN
}

(Communicated by Lance W. Small)

\begin{abstract}
In this brief note, we discuss trace methods in twisted group algebras. Specifically, we obtain information on the trace of idempotent and nilpotent elements. As is to be expected, if the ground field has positive characteristic, then the arguments used for ordinary group rings carry over to this context with little difficulty. On the other hand, lifting these results to characteristic zero algebras is not straightforward and requires a reduction to finitely presented groups.
\end{abstract}

Let $K^{t}[G]$ denote a twisted group algebra of the multiplicative group $G$ over the field $K$. Then $K^{t}[G]$ is an associative $K$-algebra with $K$-basis $\bar{G}=\{\bar{x} \mid x \in G\}$ and with multiplication defined distributively by $\bar{x} \cdot \bar{y}=\tau(x, y) \overline{x y}$ for all $x, y \in G$. Here $\tau: G \times G \rightarrow K^{\bullet}$ is the "twisting function" and, as is well known, the associative law in $K^{t}[G]$ is equivalent to the fact that $\tau$ is a 2-cocycle. Furthermore, we can always assume that $\overline{1}$ is the identity element of $K^{t}[G]$. With this assumption, the trace map tr: $K^{t}[G] \rightarrow K$ is defined linearly by $\operatorname{tr} \bar{x}=0$ if $1 \neq x \in G$ and $\operatorname{tr} \overline{1}=1$. In other words, for any element $\alpha \in K^{t}[G], \operatorname{tr} \alpha$ is the coefficient of the identity in $\alpha$. Now if $x \in G$, then $\overline{x^{-1}}$ is a scalar multiple of $(\bar{x})^{-1}$ and consequently $\overline{x^{-1}}$ and $\bar{x}$ commute. Using this, it follows that $\operatorname{tr} \alpha \beta=\operatorname{tr} \beta \alpha$ for all $\alpha, \beta \in K^{t}[G]$. Note that the ordinary group algebra $K[G]$ corresponds to the twisting function with constant value 1 .

If char $K=p>0$, we really have nothing new to offer here. Trace methods in ordinary group algebras are studied via the $p$ th power map (see [P2, Lemma 2.3.1]) and, once we know that $\operatorname{tr} \alpha \beta=\operatorname{tr} \beta \alpha$ holds in $K^{t}[G]$, the arguments carry over almost verbatim. In particular, we have the following results from [Z] and [P1]. (See also [P2, Theorem 2.3.5 and Lemma 2.3.3].)

Theorem 1. Let $K^{t}[G]$ be a twisted group algebra with char $K=p>0$.

(i) (Zalesskiu) If $e \in K^{t}[G]$ is an idempotent, then $\operatorname{tr} e \in \mathrm{GF}(p)$.

(ii) If $\alpha \in K^{t}[G]$ is nilpotent and if its support contains no nonidentity elements of p-power order, then $\operatorname{tr} \alpha=0$.

Proof. Only part (i) requires any comment. For this, write $e=\sum_{x \in S} a_{x} \bar{x}$, where $S$ is its finite support, and set $T=\{x \in S \mid x$ is a $p$-element $\}$. Since $T$ is a finite set, there exists a $p$-power $t$ so that $x^{t}=1$ for all $x \in T$, and note that $\bar{x}^{t}=k_{x} \in K^{\bullet}$

Received by the editors June 14, 1999.

2000 Mathematics Subject Classification. Primary 16S35.

This research was supported by NSF Grant DMS-9820271. The author would like to thank Prof. Jairo Z. Gonçalves for interesting conversations on this subject. 
for each such $x$. Now let $q$ be any $p$-power. Then, by [P2, Lemma 2.3.1], we have

$$
e=e^{t q}=\sum_{x \in S} a_{x}^{t q} \bar{x}^{t q}+\beta
$$

where $\beta \in K^{t}[G]$ has trace 0 . Moreover, if $x^{t q}=1$, then $x \in T$ and $\bar{x}^{t q}=\left(\bar{x}^{t}\right)^{q}=k_{x}^{q}$. Thus

$$
\operatorname{tr} e=\operatorname{tr} e^{t q}=\sum_{x \in T} a_{x}^{t q} k_{x}^{q}=\left(\sum_{x \in T} a_{x}^{t} k_{x}\right)^{q} .
$$

In particular, taking $q=p$ and $q=1$ in turn, we conclude that

$$
\operatorname{tr} e=\left(\sum_{x \in T} a_{x}^{t} k_{x}\right)^{p}=(\operatorname{tr} e)^{p}
$$

and $\operatorname{tr} e \in \mathrm{GF}(p)$, as required.

Next, we use the fact that if $I$ is an ideal of $K^{t}[G]$ with $\operatorname{tr} I=0$, then $I=0$. This, along with (ii) above, yields

Corollary 2. Let char $K=p>0$ and suppose that $G$ is a $p^{\prime}$-group, that is, a group with no elements of order $p$. Then $K^{t}[G]$ has no nonzero nil ideals.

In the case of ordinary group rings, results in characteristic 0 are usually lifted from those of characteristic $p$ by using a place argument. But for twisted group algebras, the set $\tau(G, G)=\{\tau(x, y) \mid x, y \in G\}$ can generate a very large subgroup of $K^{\bullet}$ and therefore effectively prevent the use of place maps to fields of characteristic $p$. Nevertheless, we can overcome this difficulty with the following trick.

Lemma 3. Let $K^{t}[G]$ be a twisted group algebra, let $f(\zeta) \in K[\zeta]$ be a polynomial, and suppose that $\alpha \in K^{t}[G]$ satisfies $f(\alpha)=0$. Then there exist a finitely presented group $H$, a twisted group algebra $K^{t}[H]$ over $K$, and an element $\beta \in K^{t}[H]$ such that $f(\beta)=0$ and $\operatorname{tr} \beta=\operatorname{tr} \alpha$.

Proof. Write $\alpha=a_{0}+a_{1} \overline{g_{1}}+a_{2} \overline{g_{2}}+\cdots+a_{m} \overline{g_{m}} \in K^{t}[G]$ with $g_{1}, g_{2}, \ldots, g_{m}$ distinct nonidentity elements of $G$. Then $\operatorname{tr} \alpha=a_{0}$, and without loss of generality, we can assume that the above $m$ elements generate $G$. Now let $F=F\left\langle z_{1}, z_{2}, \ldots, z_{m}\right\rangle$ be a free group on $m$ generators and suppose that $\operatorname{deg} f(\zeta)=n$. Use $W$ to denote the finite set of semigroup words in $z_{1}, z_{2}, \ldots, z_{m}$ of degree $\leq n$ and define the equivalence relation $\sim$ on $W$ by $u \sim v$ if and only if $u\left(g_{1}, g_{2}, \ldots, g_{m}\right)=v\left(g_{1}, g_{2}, \ldots, g_{m}\right)$. If $N$ is the normal subgroup of $F$ generated by all expressions of the form $u v^{-1}$ with $u, v \in W$ and $u \sim v$, then $N$ is finitely generated as a normal subgroup, so $H=F / N$ is a finitely presented group. Furthermore, $N$ maps to 1 under the natural epimorphism $F \rightarrow G$ given by $z_{i} \mapsto g_{i}$, so we obtain an epimorphism $\theta: H \rightarrow G$ with $h_{i}=z_{i} N \mapsto g_{i}$.

Next, observe that $\tau$ lifts to a 2-cocycle $\sigma: H \times H \rightarrow K^{\bullet}$ given by $\sigma(x, y)=$ $\tau(\theta(x), \theta(y))$ for all $x, y \in H$. In turn, this allows us to define a twisted group algebra $K^{t}[H]$, using $\sigma$, and then $\theta$ clearly extends to an algebra epimorphism $\theta: K^{t}[H] \rightarrow K^{t}[G]$ via $\theta(\bar{x})=\overline{\theta(x)}$ for all $x \in H$. Furthermore, if we define the element $\beta \in K^{t}[H]$ by $\beta=a_{0}+a_{1} \overline{h_{1}}+a_{2} \overline{h_{2}}+\cdots+a_{m} \overline{h_{m}}$, then $\operatorname{tr} \beta=a_{0}=\operatorname{tr} \alpha$ and $\theta(\beta)=\alpha$. Finally, since $f(\zeta) \in K[\zeta]$ is a polynomial of degree $n$, we see that

$$
f(\beta)=\sum_{w \in W} k_{w} \overline{w\left(h_{1}, h_{2}, \ldots, h_{m}\right)}
$$


for suitable $k_{w} \in K$. In particular, via the homomorphism $\theta$, we have

$$
0=f(\alpha)=\theta(f(\beta))=\sum_{w \in W} k_{w} \overline{w\left(g_{1}, g_{2}, \ldots, g_{m}\right)} .
$$

Now, in order for $f(\alpha)$ to be 0 , it is clear that we must have $\sum_{w \in \mathcal{C}} k_{w}=0$ for each equivalence class $\mathcal{C}$ under the relation $\sim$ as given above. But, by definition of the group $H$, we have $u \sim v$ if and only if $u\left(h_{1}, h_{2}, \ldots, h_{m}\right)=v\left(h_{1}, h_{2}, \ldots, h_{m}\right)$. Thus the various conditions $\sum_{w \in \mathcal{C}} k_{w}=0$ also imply that $f(\beta)=0$ and with this observation, the result follows.

Now the reason for studying finitely presented groups is that their twisting is not too large. Specifically, if $K^{t}[G]$ is given, then we say that $\bar{G} \rightarrow \widetilde{G}=\{\widetilde{g} \mid g \in G\}$ is a diagonal change of basis if we have $\widetilde{g}=d_{g} \bar{g} \in K^{\bullet} \bar{g}$ for all $g \in G$. Of course, we insist that $\widetilde{1}=1=\overline{1}$, so $d_{1}=1$. Obviously, this procedure changes $\tau$ by a factor which is a 2-coboundary. The following is presumably well known.

Lemma 4. Let $K^{t}[G]$ be a twisted group ring with $G$ a finitely presented group. Then there exists a diagonal change of basis such that if $\tau: G \times G \rightarrow K^{\bullet}$ is the new twisting, then $\tau(G, G)$ is contained in a finitely generated subgroup of $K^{\bullet}$.

Proof. Let $K^{\bullet} \bar{G}$ be the group of trivial units in $K^{t}[G]$. Then the map $\nu: K^{\bullet} \bar{G} \rightarrow G$ given by $k \bar{g} \mapsto g$ is an epimorphism. In particular, since $G$ is finitely generated, there exists a finitely generated subgroup $\mathcal{G}$ of $K^{\bullet} \bar{G}$ such that $\nu: \mathcal{G} \rightarrow G$ is onto. Of course, the kernel here is $\mathcal{G} \cap K^{\bullet} \triangleleft \mathcal{G}$ and, since $G$ is finitely presented, [P2, Lemma 12.3.12(ii)] implies that $\mathcal{G} \cap K^{\bullet}$ is finitely generated as a normal subgroup of $\mathcal{G}$. But $\mathcal{G} \cap K^{\bullet}$ is central, so it must be a finitely generated abelian group. Finally, let $\widetilde{G}$ be a transversal for $\mathcal{G} \cap K^{\bullet}$ in $\mathcal{G}$. Then $\widetilde{G}$ is clearly a new basis for $K^{t}[G]$ and $\bar{G} \rightarrow \widetilde{G}$ is a diagonal change of basis. Furthermore, if $\tau$ is the new twisting function, then $\tau(G, G) \subseteq \mathcal{G} \cap K^{\bullet}$, and the result follows.

Now suppose that char $K=0$ and that $G$ and $\tau$ are as in the preceding lemma. If $\alpha \in K^{t}[G]$, then there exists a finitely generated subring $R$ of $K$ such that the coefficients of $\alpha$ and the subgroup of $K^{\bullet}$ generated by $\tau(G, G)$ are all in $R$. Then $R \bar{G}$ is a subring of $K^{t}[G]$ containing $\alpha$. Furthermore, $R \bar{G}$ is clearly a twisted group ring which we denote by $R^{t}[G]$. Note that, if $L$ is a field of characteristic 0 or $p$ and if $\lambda: R \rightarrow L$ is a ring homomorphism, then $\lambda$ extends to a map $\lambda: R^{t}[G] \rightarrow L^{t}[G]$, where the latter is some twisted group algebra of $G$ over $L$. Of course, if $f(\alpha)=0$ and if $R$ contains all coefficients of the polynomial $f(\zeta) \in K[\zeta]$, then $\lambda(\alpha)$ satisfies the polynomial $(\lambda f)(\zeta) \in L[\zeta]$. Consequently, we can use the usual place arguments to lift characteristic $p$ results to characteristic 0 fields.

Theorem 5. Let $K^{t}[G]$ be a twisted group algebra with char $K=0$.

(i) (Zalesskiü) If $e \in K^{t}[G]$ is an idempotent, then tre is rational.

(ii) If $\alpha \in K^{t}[G]$ is nilpotent, then $\operatorname{tr} \alpha=0$.

Proof. In view of Lemma 3 , it suffices to assume that $G$ is a finitely presented group, and then by Lemma 4 , we can assume that $\tau(G, G)$ is contained in a finitely generated subgroup of $K^{\bullet}$. With this observation and the above remarks, we can now apply the place and number-theoretic arguments of [Z] (see [P2, Theorem 2.3.5 and Lemma 2.3.3]) to obtain the results.

Corollary 6. Let char $K=0$; then $K^{t}[G]$ has no nonzero nil ideals. 
Furthermore, the proof of [P2, Theorem 2.3.8] yields

Theorem 7. Let $\alpha$ be an algebraic element of the twisted group algebra $K^{t}[G]$ with minimal polynomial $f(\zeta) \in K[\zeta]$, and let $\lambda_{1}, \lambda_{2}, \ldots, \lambda_{n}$ be the distinct roots of $f$ in some algebraic closure of $K$.

(i) If char $K=0$, then there exist rational numbers $r_{1}, r_{2}, \ldots, r_{n}$ satisfying the relations $r_{1}+r_{2}+\cdots+r_{n}=1$ and $\operatorname{tr} \alpha=r_{1} \lambda_{1}+r_{2} \lambda_{2}+\cdots+r_{n} \lambda_{n}$.

(ii) If char $K=p>0$ and if either $G$ is a $p^{\prime}$-group or $f(\zeta)$ has no multiple roots, then there exist $r_{1}, r_{2}, \ldots, r_{n} \in G F(p)$ with $r_{1}+r_{2}+\cdots+r_{n}=1$ and $\operatorname{tr} \alpha=r_{1} \lambda_{1}+r_{2} \lambda_{2}+\cdots+r_{n} \lambda_{n}$.

Finally, it would be interesting to know whether Kaplansky's theorem [K] also holds in this context. Namely, if $e$ is an idempotent in $K^{t}[G]$ and if $\operatorname{char} K=0$, is it true that $\operatorname{tr} e \geq 0$ ? This certainly holds when $G$ is finite and also when $\tau(G, G)$ consists of complex numbers of absolute value 1 . But, to the author's knowledge, the general case is still open.

\section{REFERENCES}

[K] I. Kaplansky, Fields and Rings, Chicago Lectures in Math., Univ. of Chicago Press, Chicago, 1969. MR 42:4345

[P1] D. S. Passman, Radicals of twisted group rings, Proc. London Math. Soc. (3) 20 (1970), 409-437. MR 42:6129

[P2] _ The Algebraic Structure of Group Rings, Wiley-Interscience, New York, 1977. MR 81d:16001

[Z] A. E. Zalesskiǔ, On a problem of Kaplansky, Soviet Math. 13 (1972), 449-452. MR 45:6947

Department of Mathematics, University of Wisconsin, Madison, Wisconsin 53706

E-mail address: passman@math.wisc.edu 